\title{
A Three Dimensional Wave Activity Flux Applicable to Inertio-Gravity Waves
}

\author{
Saburo Miyahara \\ Department of Earth and Planetary Sciences, Kyushu University, Fukuoka, Japan
}

\begin{abstract}
A three dimensional wave activity flux applicable to non-hydrostatic inertio-gravity waves in a time mean flow in a Boussinesq fluid is derived. It is shown that the flux gives the wave-action density flux relative to the local time mean flow, and additive terms in residual circulation are equal to Stokes drift under the WKB limit. The flux is a generalization of three dimensional wave activity fluxes that have been used to analyze quasi-geostrophic wave disturbances. A flux under the log-pressure coordinate system on spherical geometry applicable to global hydrostatic inertio-gravity waves is also derived.
\end{abstract}

\section{Introduction}

The Eliassen-Palm (E-P) flux is widely used to analyze wave activities and propagation in meridional plane in zonal mean zonal winds (e.g., Andrews and McIntyre 1976; Andrews et al. 1987). The E-P flux analysis is extended to three dimensional fluxes that are applicable to quasi-geostrophic disturbances propagating in three dimensional space (e.g., Plumb 1985, 1986; Trenberth 1986; Takaya and Nakamura 2001). These fluxes are useful to analyze three dimensional propagation of quasi-geostrophic disturbances, but the applicability is limited to these disturbances. In recent years, global distributions of gravity waves are revealed observationally and numerically (e.g., Sato et al. 1999; Tsuda et al. 2000; Kawatani et al. 2003). It therefore seems desirable to define a three dimensional wave activity flux that is applicable to gravity waves for analyzing three dimensional propagation of gravity waves. A general method to define an exact conservation law of wave activity is constructed (McIntyre and Shepherd 1987; Haynes 1988), but it seems to be not easy to apply actual wave disturbances (Haynes 1988) in contrast to the E-P flux and the quasi-geostrophic three dimensional fluxes.

In this article, a three dimensional wave activity flux applicable to non-hydrostatic inertio-gravity waves in a time mean flow in a Boussinesq fluid is derived. It is shown that the flux gives the wave-action density flux relative to the local time mean flow under the WKB limit.

The derivation of the flux is outlined and its physical meaning is discussed in Section 2. A flux under the log pressure coordinate system on spherical geometry is derived in Section 3. Section 4 is devoted to conclusions.

\section{Three dimensional wave activity flux in a Boussinesq fluid}

In this section, the derivation of three dimensional wave activity flux in a Boussinesq fluid on beta-plane is outlined, and the physical meaning of the flux is examined under the WKB limit (weak shear limit of time mean background winds).

We consider a Boussinesq fluid on beta-plane, and we put $u=\bar{u}+u^{\prime}$, etc. for all the physical variables, where the overbar denotes a time mean. The time mean equations for the mean flow including second order effects of disturbances are given as:

$$
\begin{aligned}
& \frac{D \bar{u}}{D t}-f \bar{v}=-\frac{\partial \bar{\Phi}}{\partial x}-\frac{\partial \overline{u^{\prime} u^{\prime}}}{\partial x}-\frac{\partial \overline{u^{\prime} v^{\prime}}}{\partial y}-\frac{\partial \overline{u^{\prime} w^{\prime}}}{\partial z}, \\
& \frac{D \bar{v}}{D t}+f \bar{u}=-\frac{\partial \bar{\Phi}}{\partial y}-\frac{\partial \overline{v^{\prime} u^{\prime}}}{\partial x}-\frac{\partial \overline{v^{\prime} v^{\prime}}}{\partial y}-\frac{\partial \overline{v^{\prime} w^{\prime}}}{\partial z} \\
& \frac{D \bar{r}}{D t}-N^{2} \bar{w}=-\frac{\partial \overline{r^{\prime} u^{\prime}}}{\partial x}-\frac{\partial \overline{r^{\prime} v^{\prime}}}{\partial y}-\frac{\partial \overline{r^{\prime} w^{\prime}}}{\partial z}
\end{aligned}
$$

where

$$
\frac{D}{D t}=\frac{\partial}{\partial t}+\bar{u} \frac{\partial}{\partial x}+\bar{v} \frac{\partial}{\partial y}+\bar{w} \frac{\partial}{\partial z}
$$

and $\bar{u}, \bar{v}$, and $\bar{w}$ are the background time mean zonal, meridional, and vertical flow, respectively, $N^{2}$ is the static stability, and $r$ is the buoyancy. Note that the time derivative is retained in the equation system to denote the time variation with longer time scale than the time mean. Other notations are conventional. If we define the difference between the kinetic energy and the available potential energy

$\bar{S} \equiv \frac{1}{2}\left(\overline{u^{\prime 2}}+\overline{v^{\prime 2}}+\overline{w^{\prime 2}}-\frac{\overline{r^{\prime 2}}}{N^{2}}\right)$,

the time mean equations are rewritten as follows:

$$
\begin{aligned}
\frac{D \bar{u}}{D t}-f \bar{v}= & -\frac{\partial \bar{\Phi}}{\partial x}-\frac{\partial \bar{S}}{\partial x}-\frac{\partial}{\partial x} \frac{1}{2}\left(\overline{u^{\prime 2}}-\overline{v^{\prime 2}}-\overline{w^{\prime 2}}+\frac{\overline{r^{\prime 2}}}{N^{2}}\right) \\
& -\frac{\partial \overline{u^{\prime} v^{\prime}}}{\partial y}-\frac{\partial \overline{u^{\prime} w^{\prime}}}{\partial z}, \\
\frac{D \bar{v}}{D t}+f \bar{u}= & -\frac{\partial \bar{\Phi}}{\partial y}-\frac{\partial \bar{S}}{\partial y}-\frac{\partial \overline{v^{\prime} u^{\prime}}}{\partial x}-\frac{\partial}{\partial y} \frac{1}{2}\left(\overline{v^{\prime 2}}-\overline{u^{\prime 2}}-\overline{w^{\prime 2}}+\frac{\overline{r^{\prime 2}}}{N^{2}}\right) \\
& -\frac{\partial \overline{v^{\prime} w^{\prime}}}{\partial z}, \\
\frac{D \bar{r}}{D t}-N^{2} \bar{w}= & -\frac{\partial \overline{r^{\prime} u^{\prime}}}{\partial x}-\frac{\partial \overline{r^{\prime} v^{\prime}}}{\partial y}-\frac{\partial \overline{r^{\prime} w^{\prime}}}{\partial z} .
\end{aligned}
$$

Corresponding author: Saburo Miyahara, Department of Earth and Planetary Sciences, Kyushu University, 6-10-1 Hakozaki, Fukuoka 812-8581, Japan. E-mail: sbm@geo.kyushu-u.ac.jp. (C2006, the Meteorological Society of Japan. 
This equation system is transformed to the equation system that is analogous to the transformed Eulerianmean (TEM) equation system

$$
\begin{aligned}
& \frac{D \bar{u}}{D t}-f \bar{v}^{*}=-\frac{\partial \bar{\Phi}}{\partial x}-(\nabla \cdot F)_{x}, \\
& \frac{D \bar{v}}{D t}+f \bar{u}^{*}=-\frac{\partial \bar{\Phi}}{\partial y}-(\nabla \cdot F)_{y}, \\
& \frac{D \bar{r}}{D t}-N^{2} \bar{w}^{*}=-\frac{\partial \overline{r^{\prime} w^{\prime}}}{\partial z},
\end{aligned}
$$

where $\bar{u}^{*}, \bar{v}^{*}$, and $\bar{w}^{*}$ are the residual circulation defined by (7), and $F$ is the tensor defined by (8).

This equation system is a three dimensional extension of the TEM equation system, and also an extension of the three dimensional systems by Plumb (1986) to non-geostrophic and non-hydrostatic disturbances. Note that the residual circulation does not satisfy the continuity equation, because of the presence of beta-effect.

$$
\left.\begin{array}{l}
\hat{C}_{g x}=\frac{\partial \hat{\omega}}{\partial k}=C_{g x}-\bar{u}=\frac{k}{\hat{\omega}} \frac{N^{2}-\hat{\omega}^{2}}{k^{2}+l^{2}+m^{2}} \\
\hat{C}_{g y}=\frac{\partial \hat{\omega}}{\partial l}=C_{g y}-\bar{v}=\frac{l}{\hat{\omega}} \frac{N^{2}-\hat{\omega}^{2}}{k^{2}+l^{2}+m^{2}} \\
\hat{C}_{g z}=\frac{\partial \hat{\omega}}{\partial m}=C_{g z}-\bar{w}=\frac{m}{\hat{\omega}} \frac{f^{2}-\hat{\omega}^{2}}{k^{2}+l^{2}+m^{2}}
\end{array}\right\}
$$

By utilizing $\overline{a^{\prime} b^{\prime}}=\frac{1}{4}\left(\mathrm{AB}^{*}+\mathrm{A}^{*} \mathrm{~B}\right)$, where ${ }^{*}$ denotes the complex conjugate, we obtain

$E=\frac{1}{2}\left(\overline{u^{\prime 2}}+\overline{v^{\prime 2}}+\overline{w^{\prime 2}}+\frac{\overline{r^{\prime 2}}}{N^{2}}\right)=\frac{1}{2} \frac{\left(k^{2}+l^{2}\right)\left(f^{2}-N^{2}\right) \hat{\omega}^{2}}{\left(\hat{\omega}^{2}-f^{2}\right)^{2}\left(\hat{\omega}^{2}-N^{2}\right)}|\Phi|^{2}$,

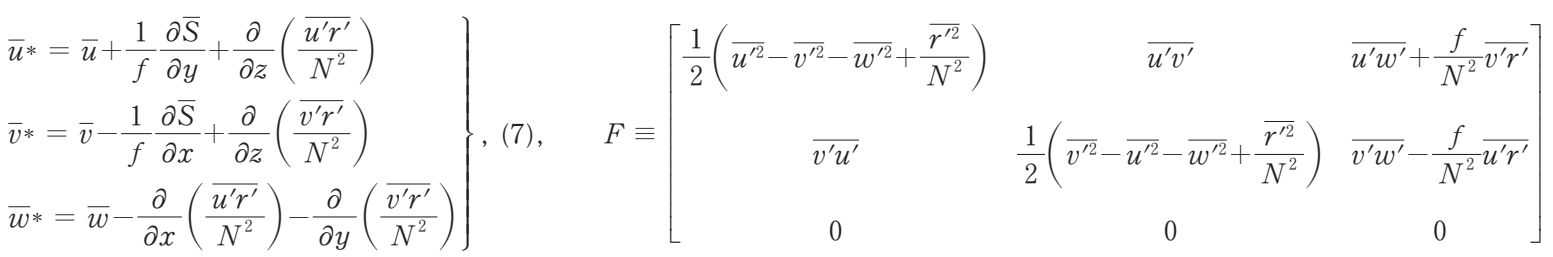

To investigate the physical meaning of the tensor $F$ and the residual mean circulation, we consider a wave packet of inertio-gravity wave disturbance in a time mean flow. Here, we consider a weak shear limit of the background time mean flows $\bar{u}, \bar{v}$, and $\bar{w}$, and we treat the wave disturbance as a plane wave under the WKB limit. In this case a physical quantity of the wave disturbance is written as follows:

$a^{\prime}(x, y, z, t)=A \exp \{i(k x+l y+m z-\omega t)\}$,

where $A$ denotes the slowly varying amplitude of the wave packet. The perturbation equations of the amplitudes under the WKB limit are given by

$$
\left.\begin{array}{rl}
-i \hat{\omega} U-f V & =-i k \Phi \\
-i \hat{\omega} V+f U & =-i l \Phi \\
-i \hat{\omega} W & =-i m \Phi-R \\
-i \hat{\omega} R-N^{2} W & =0 \\
i k U+i l V+i m W & =0
\end{array}\right\},
$$

where $\hat{\omega}=\omega-k \bar{u}-l \bar{v}-m \bar{w}$ is the intrinsic frequency. By solving the simultaneous equation, we obtain following local dispersion relation and the wave amplitudes of the inertio-gravity wave.

$\hat{\omega}^{2}=\frac{\left(k^{2}+l^{2}\right) N^{2}+f^{2} m^{2}}{k^{2}+l^{2}+m^{2}}$,

$U=\frac{k \hat{\omega}+i l f}{\hat{\omega}^{2}-f^{2}} \Phi, \quad V=\frac{l \hat{\omega}-i k f}{\hat{\omega}^{2}-f^{2}} \Phi$,

$W=\frac{m \hat{\omega}}{\hat{\omega}^{2}-N^{2}} \Phi, \quad R=\frac{i m N^{2}}{\hat{\omega}^{2}-N^{2}} \Phi$.

We define the intrinsic group velocity $\widehat{\mathbf{C}}_{g}$ and the phase velocity $\hat{C}$ defined by using the intrinsic frequency as follows:

$$
\begin{aligned}
& \hat{C}_{g x} E=\frac{1}{2} \frac{k \hat{\omega}}{\hat{\omega}^{2}-f^{2}}|\Phi|^{2}, \\
& \hat{C}_{g y} E=\frac{1}{2} \frac{l \hat{\omega}}{\hat{\omega}^{2}-f^{2}}|\Phi|^{2}, \\
& \hat{C}_{g z} E=\frac{1}{2} \frac{m \hat{\omega}}{\hat{\omega}^{2}-N^{2}}|\Phi|^{2},
\end{aligned}
$$

and

$$
\begin{aligned}
& \frac{1}{2}\left(\overline{u^{\prime 2}}-\overline{v^{\prime 2}}-\overline{w^{\prime 2}}+\frac{\overline{r^{\prime 2}}}{N^{2}}\right)=\frac{1}{2} \frac{k \hat{\omega}}{\hat{\omega}^{2}-f^{2}}|\Phi|^{2} \frac{k}{\hat{\omega}}=\hat{C}_{g x} \frac{E}{\hat{C}_{x}} \\
& \frac{1}{2}\left(\overline{v^{\prime 2}}-\overline{u^{\prime 2}}-\overline{w^{\prime 2}}+\frac{\overline{r^{\prime 2}}}{N^{2}}\right)=\frac{1}{2} \frac{l \hat{\omega}}{\hat{\omega}^{2}-f^{2}}|\Phi|^{2} \frac{l}{\hat{\omega}}=\hat{C}_{g y} \frac{E}{\hat{C}_{y}} \\
& \overline{u^{\prime} w^{\prime}}+\frac{f}{N^{2}} \overline{v^{\prime} r^{\prime}}=\frac{1}{\hat{\omega}^{2}-f^{2}}\left(k \hat{\omega}-\frac{k f^{2}}{\hat{\omega}}\right) \hat{C}_{g z} E=\hat{C}_{g z} \frac{E}{\hat{C}_{x}} \\
& \overline{v^{\prime} w^{\prime}}-\frac{f}{N^{2}} \overline{u^{\prime} r^{\prime}}=\frac{1}{\hat{\omega}^{2}-f^{2}}\left(l \hat{\omega}-\frac{l f^{2}}{\hat{\omega}}\right) \hat{C}_{g z} E=\hat{C}_{g z} \frac{E}{\hat{C}_{y}} \\
& \overline{u^{\prime} v^{\prime}}=\frac{1}{2} \frac{k l}{\hat{\omega}^{2}-f^{2}}|\Phi|^{2}=\hat{C}_{g x} \frac{E}{\hat{C}_{y}}=\hat{C}_{g y} \frac{E}{\hat{C}_{x}}
\end{aligned}
$$

and $F$ is denoted using (14) as follows:

$F \equiv\left[\begin{array}{ccc}\hat{C}_{g x} \frac{E}{\hat{C}_{x}} & \hat{C}_{g y} \frac{E}{\hat{C}_{x}} & \hat{C}_{g z} \frac{E}{\hat{C}_{x}} \\ \hat{C}_{g x} \frac{E}{\hat{C}_{y}} & \hat{C}_{g y} \frac{E}{\hat{C}_{y}} & \hat{C}_{g z} \frac{E}{\hat{C}_{y}} \\ 0 & 0 & 0\end{array}\right]$

As shown in (15) the flux tensor $F$ gives the wave-action density flux relative to the time mean flow under the WKB limit.

Next we consider the physical meaning of the residual circulation defined by (7). It is shown that the 
additional terms in (7) are

$$
\left.\begin{array}{rl}
\frac{1}{f} & \frac{\partial \bar{S}}{\partial y}+\frac{\partial}{\partial z}\left(\frac{\overline{u^{\prime} r^{\prime}}}{N^{2}}\right) \\
& =\frac{1}{2}\left\{\frac{f\left(k^{2}+l^{2}\right)}{\left(\hat{\omega}^{2}-f^{2}\right)^{2}} \frac{\partial|\Phi|^{2}}{\partial y}+\frac{m l f}{\left(\hat{\omega}^{2}-N^{2}\right)\left(\hat{\omega}^{2}-f^{2}\right)} \frac{\partial|\Phi|^{2}}{\partial z}\right\} \\
- & \frac{1}{f} \frac{\partial \bar{S}}{\partial x}+\frac{\partial}{\partial z}\left(\frac{\overline{v^{\prime} r^{\prime}}}{N^{2}}\right) \\
& =-\frac{1}{2}\left\{\frac{f\left(k^{2}+l^{2}\right)}{\left(\hat{\omega}^{2}-f^{2}\right)^{2}} \frac{\partial|\Phi|^{2}}{\partial x}+\frac{m k f}{\left(\hat{\omega}^{2}-N^{2}\right)\left(\hat{\omega}^{2}-f^{2}\right)} \frac{\partial|\Phi|^{2}}{\partial z}\right\} \\
- & \frac{\partial}{\partial x}\left(\frac{\overline{u^{\prime} r^{\prime}}}{N^{2}}\right)-\frac{\partial}{\partial y}\left(\frac{\overline{v^{\prime} r^{\prime}}}{N^{2}}\right) \\
& =-\frac{1}{2} \frac{m f}{\left(\hat{\omega}^{2}-N^{2}\right)\left(\hat{\omega}^{2}-f^{2}\right)}\left(l \frac{\partial|\Phi|^{2}}{\partial x}-k \frac{\partial|\Phi|^{2}}{\partial y}\right)
\end{array}\right\}
$$

where the derivatives of $\hat{\omega}$ and $f$ are neglected because we assume that the spatial variations of the amplitudes are faster than the variations of $\hat{\omega}$ and $f$. On the other hand, it is shown that the Stokes drifts are given by

$$
\begin{aligned}
& \bar{u}_{s}=\overline{\xi^{\prime} \frac{\partial u^{\prime}}{\partial x}}+\overline{\eta^{\prime} \frac{\partial u^{\prime}}{\partial y}}+\overline{\overline{\zeta^{\prime} \frac{\partial u^{\prime}}{\partial z}}}=\frac{\partial \overline{\xi^{\prime} u^{\prime}}}{\partial x}+\frac{\partial \overline{\eta^{\prime} u^{\prime}}}{\partial y}+\frac{\partial \overline{\zeta^{\prime} u^{\prime}}}{\partial z} \\
& =\frac{1}{2}\left\{\frac{f\left(k^{2}+l^{2}\right)}{\left(\hat{\omega}^{2}-f^{2}\right)^{2}} \frac{\partial|\Phi|^{2}}{\partial y}+\frac{m l f}{\left(\hat{\omega}^{2}-N^{2}\right)\left(\hat{\omega}^{2}-f^{2}\right)} \frac{\partial|\Phi|^{2}}{\partial z}\right\}, \\
& \bar{v}_{s}=\overline{\xi^{\prime} \frac{\partial v^{\prime}}{\partial x}}+\overline{\eta^{\prime} \frac{\partial v^{\prime}}{\partial y}}+\overline{\zeta^{\prime} \frac{\partial v^{\prime}}{\partial z}}=\frac{\partial \overline{\xi^{\prime} v^{\prime}}}{\partial x}+\frac{\partial \overline{\eta^{\prime} v^{\prime}}}{\partial y}+\frac{\partial \overline{\zeta^{\prime} v^{\prime}}}{\partial z} \\
& =-\frac{1}{2}\left\{\frac{f\left(k^{2}+l^{2}\right)}{\left(\hat{\omega}^{2}-f^{2}\right)^{2}} \frac{\partial|\Phi|^{2}}{\partial x}+\frac{m k f}{\left(\hat{\omega}^{2}-N^{2}\right)\left(\hat{\omega}^{2}-f^{2}\right)} \frac{\partial|\Phi|^{2}}{\partial z}\right\}, \\
& \bar{w}_{s}=\overline{\xi^{\prime} \frac{\partial w^{\prime}}{\partial x}}+\overline{\eta^{\prime} \frac{\partial w^{\prime}}{\partial y}}+\overline{\zeta^{\prime} \frac{\partial w^{\prime}}{\partial z}}=\frac{\partial \overline{\xi^{\prime} w^{\prime}}}{\partial x}+\frac{\partial \overline{\eta^{\prime} w^{\prime}}}{\partial y}+\frac{\partial \overline{\zeta^{\prime} w^{\prime}}}{\partial z} \\
& =-\frac{1}{2} \frac{m f}{\left(\hat{\omega}^{2}-N^{2}\right)\left(\hat{\omega}^{2}-f^{2}\right)}\left(l \frac{\partial|\Phi|^{2}}{\partial x}-k \frac{\partial|\Phi|^{2}}{\partial y}\right),
\end{aligned}
$$

where $\xi^{\prime}, \eta^{\prime}$, and $\zeta^{\prime}$ are the displacement in $x, y$, and $z$ directions, respectively, and satisfy the continuity equation;

$\frac{\partial \xi^{\prime}}{\partial x}+\frac{\partial \eta^{\prime}}{\partial y}+\frac{\partial \zeta^{\prime}}{\partial z}=0$

To derive (17) we also neglect the derivatives of $\hat{\omega}$ and $f$, and we assume the WKB limit that $\xi^{\prime}, \eta^{\prime}$, and $\zeta^{\prime}$ are given by

$\xi^{\prime}=\frac{i}{\hat{\omega}} u^{\prime}, \quad \eta^{\prime}=\frac{i}{\hat{\omega}} v^{\prime}, \quad$ and $\quad \zeta^{\prime}=\frac{i}{\hat{\omega}} w^{\prime}$

The approximation (18) is valid in the weak shear limit of the time mean flow (Andrews et al. 1987). Equations (16) and (17) show that the additional terms in the residual circulation are equal to the Stokes drifts under the WKB limit, and it is also a three dimensional extension of the residual mean meridional circulation used in the TEM equation system (Andrews et al. 1987).

Similar results can be obtained for inertio-gravity waves under the hydrostatic approximation by neglecting the square terms of vertical velocity perturbation in $E, F$, and $\bar{S}$.

\section{Wave disturbances in the log-pressure co- ordinate system on spherical geometry}

It is not difficult to apply the results of hydrostatic case obtained in Section 2 to wave disturbances in the log-pressure coordinate system on spherical geometry. In this case the transformed time mean equations become;

$\frac{D \bar{u}}{D t}-\frac{\bar{u} \tan \phi}{a} \bar{v}-2 \Omega \sin \phi \bar{v}^{*}=-\frac{1}{a \cos \phi} \frac{\partial \bar{\Phi}}{\partial \lambda}-(p a \cos \phi)^{-1}(\nabla \cdot F)_{\lambda}$,

$\frac{D \bar{v}}{D t}+\frac{\bar{u} \tan \phi}{a} \bar{u}+2 \Omega \sin \phi \bar{u}^{*}=-\frac{1}{a} \frac{\partial \bar{\Phi}}{\partial \phi}-(p a \cos \phi)^{-1}(\nabla \cdot F)_{\phi}$,

$\frac{D \bar{\Phi}_{z}}{D t}+N^{2} \bar{w}^{*}=-\frac{1}{p} \frac{\partial \overline{\Phi_{z}^{\prime} w^{\prime}}}{\partial z}$,

where $\bar{\Phi}$ denotes the geopotential, $\Omega$ the earth's rotation rate, $\lambda$ and $\phi$ the longitude and the latitude, respectively, and note that the divergence is

$\nabla \cdot(\quad)=\frac{1}{a \cos \phi} \frac{\partial(\quad)}{\partial \lambda}+\frac{1}{a \cos \phi} \frac{\partial(\cos \phi)}{\partial \phi}+\frac{\partial(\quad)}{\partial z}$,

and the time derivative is given by

$\frac{D}{D t}=\frac{\partial}{\partial t}+\frac{\bar{u}}{a \cos \phi} \frac{\partial}{\partial \lambda}+\frac{\bar{v}}{a} \frac{\partial}{\partial \phi}+\bar{w} \frac{\partial}{\partial z}$.

In this case $F$ and $\bar{S}$ are given by (20) and (21), respectively, and the residual circulation is given by

$$
\left.\begin{array}{l}
\bar{u}^{*}=\bar{u}+\frac{1}{2 \Omega \sin \phi} \frac{1}{a \cos ^{2} \phi} \frac{\partial \bar{S} \cos ^{2} \phi}{\partial \phi}-\frac{1}{p} \frac{\partial}{\partial z}\left(\frac{p \overline{u^{\prime} \Phi_{z}^{\prime}}}{N^{2}}\right) \\
\bar{v}^{*}=\bar{v}-\frac{1}{2 \Omega \sin \phi} \frac{1}{a \cos ^{2} \phi} \frac{\partial \bar{S} \cos \phi}{\partial \lambda}-\frac{1}{p} \frac{\partial}{\partial z}\left(\frac{p \overline{v^{\prime} \Phi_{z}^{\prime}}}{N^{2}}\right) \\
\bar{w}^{*}=\bar{w}+\frac{1}{a \cos \phi} \frac{\partial}{\partial \lambda}\left(\frac{\overline{u^{\prime} \Phi_{z}^{\prime}}}{N^{2}}\right)+\frac{1}{a \cos \phi} \frac{\partial}{\partial \phi}\left(\frac{\overline{v^{\prime} \Phi_{z}^{\prime}}}{N^{2}} \cos \phi\right)
\end{array}\right\},
$$

where the residual circulation does not satisfy the continuity equation because of the latitudinal variation of the Coriolis factor and the geometric factor $\cos \phi$ included in $\bar{u}^{*}$ and $\bar{v}^{*}$. This equation system is different from the equation system derived by Trenberth (1986). The present system contains extra terms related to the available potential energy in diagonal terms of $F$ and $\bar{S}$, which are essential to obtain physically meaningful results for non-geostrophic disturbances as shown in Section 2.

In this case we also consider an inertio-gravity wave packet in a time mean flow. Assuming a weak shear

$$
F \equiv\left[\begin{array}{ccc}
\frac{1}{2}\left(\overline{u^{\prime 2}}-\overline{v^{\prime 2}}+\frac{\overline{\Phi_{z}^{\prime 2}}}{N^{2}}\right) & \overline{u^{\prime} v^{\prime}} & \overline{u^{\prime} w^{\prime}}-\frac{2 \Omega \sin \phi}{N^{2}} \overline{v^{\prime} \Phi_{z}^{\prime}} \\
\overline{v^{\prime} u^{\prime}} & \frac{1}{2}\left(\overline{v^{\prime 2}}-\overline{u^{\prime 2}}+\frac{\overline{\Phi_{z}^{\prime 2}}}{N^{2}}\right) & \overline{v^{\prime} w^{\prime}}+\frac{2 \Omega \sin \phi}{N^{2}} \overline{u^{\prime} \Phi_{z}^{\prime}} \\
0 & 0 & 0
\end{array}\right] p a \cos \phi, \quad(20), \quad \bar{S}=\frac{1}{2}\left(\overline{u^{\prime 2}}+\overline{v^{\prime 2}}-\frac{\overline{\Phi_{z}^{\prime 2}}}{N^{2}}\right)
$$


limit and considering a localized wave packet, we put a physical quantity of the wave disturbance as follows:

$a^{\prime}(\lambda, \phi, z, t)=A \exp \left\{\frac{z}{2 H}+i(s \lambda+n \phi+m z-\omega t)\right\}$,

where $H$ is a mean scale height and $A$ denotes the slowly varying amplitude of the wave packet. In this case the local intrinsic frequency is defined by $\hat{\omega}=\omega-k \bar{u}-l \bar{v}$ $-m \bar{w}$, where the local horizontal wavenumbers are given by

$k \equiv \frac{s}{a \cos \phi} \quad$ and $\quad l \equiv \frac{n}{a}$.

Ignoring the shear of the background winds and the variation of the geometric factor $\cos \phi$ with latitude, we obtain following local dispersion relation and the wave amplitudes of the inertio-gravity wave.

$\hat{\omega}^{2}=\frac{\left(k^{2}+l^{2}\right) N^{2}+f^{2}\left(m^{2}+\frac{1}{4 H^{2}}\right)}{m^{2}+\frac{1}{4 H^{2}}}$,

$U=\frac{k \hat{\omega}+i l f}{\hat{\omega}^{2}-f^{2}} \Phi, \quad V=\frac{l \hat{\omega}-i k f}{\hat{\omega}^{2}-f^{2}} \Phi, \quad W=\frac{\left(\frac{i}{2 H}-m\right) \hat{\omega}}{N^{2}} \Phi$,

where $f \equiv 2 \Omega \sin \phi$. Using the dispersion relation and the wave solution, we can show that the three dimensional flux tensor $F$ and the additional terms in the residual circulation give the three dimensional wave-action density flux relative to the local time mean flow and the Stokes drift, respectively under the WKB limit as in case of the Boussinesq fluid shown in Section 2.

\section{Conclusions}

I have shown that the three dimensional flux defined by (8) or (20) gives the three dimensional wave-action density flux relative to a time mean flow, which is applicable to inertio-gravity waves, and also shown that the residual circulation defined by (7) or (22) gives the time mean flow including the effects of Stokes drift under the WKB limit. This is a three dimensional extension of the E-P flux and the TEM equation system, and also an extension of the three dimensional fluxes given by Plumb (1986) to non-geostrophic disturbances. It might be useful to analyze three dimensional behavior of non- geostrophic wave disturbances such as inertio-gravity waves, tidal waves, and equatorial waves in a time mean flow.

Effects of shear of time mean flow, which are neglected in the present paper, remain to be investigated.

\section{Acknowledgments}

I thank Dr. M. Takahashi and an anonymous referee for comments on earlier version of this work.

\section{References}

Andrews, D. G., and M. E. McIntyre, 1976: Planetary waves in horizontal and vertical shear: the generalized EliassenPalm relation and the zonal mean acceleration. J. Atmos. Sci., 33, 2031-2048.

Andrews, D. G., J. R. Holton, and C. B. Leovy, 1987: Middle Atmosphere Dynamics, 489pp, Academic Press.

Haynes, P. H., 1988: Forced, dissipative generalizations of finiteamplitude wave-activity conservation relations for zonal and nonzonal basic flows. J. Atmos. Sci., 45, 2352-2362.

Kawatani, Y., S. K. Dhaka, M. Takahashi, and T. Tsuda, 2003: Large potential energy of gravity waves over a smooth surface with little convection: Simulation and observation, Geophys. Res. Lett., doi:10.1029/2003GL016960.

McIntyre, M. E., and T. G. Shepherd, 1987: An exact local conservation theorem for finite amplitude disturbances to non-parallel shear flows, with remarks on Hamiltonian structure and Arnol'd's stability theorems. J. Fluid Mech., $181,527-565$.

Plumb, R. A., 1985: On the three-dimensional propagation of stationary waves. J. Atmos. Sci., 42, 217-229.

Plumb, R. A., 1986: Three-dimensional propagation of transient quasi-geostrophic eddies and its relationship with eddy forcing on the time-mean flow. J. Atmos. Sci., 43, 16571678.

Sato, K., T. Kumakura, and M. Takahashi, 1999: Gravity waves appearing in a high-resolution GCM simulation. J. Atmos. Sci., 56, 1005-1018.

Takaya, K., and H. Nakamura, 2001: A formulation of a phaseindependent wave-activity flux for stationary and migratory quasigeostrophic eddies on a zonally varying basic flow. J. Atmos. Sci., 58, 608-627.

Trenberth, K. E., 1986: An assessment of the impact of transient eddies on the zonal flow during a blocking episode using localized Eliassen-Palm flux diagnostics. J. Atmos. Sci., 43, 2070-2087.

Tsuda, T., M. Nishida, C. Rocken, and R. H. Ware, 2000: A global morphology of gravity wave activity in the stratosphere revealed by the GPS occultation data (GPS/MET). J. Geophys. Res., 105, 7257-7273.

Manuscript received 15 May 2006, accepted 19 June 2006 SOLA: http://www.jstage.jst.go.jp/browse/sola/ 